\title{
Capturing Alloreactivity in Multiple Myeloma
}

\author{
Gordon Cook, MBChB, PhD, FRCP, FRCPath, FRCPI \\ Director, Blood and Marrow Transplantation Programme, Leeds Teaching Hospitals Trust, and \\ Consultant Haematologist and Honorary Senior Lecturer, University of Leeds
}

\begin{abstract}
Allogeneic stem cell transplantation (alloSCT) has been utilized in the management of both malignant and non-malignant hematological disorders for several decades and has established its role in producing long-term remissions. In the context of multiple myeloma (MM), compared with conventional therapies alloSCT induces the highest rate of remissions, resulting in long-term disease-free survival in over $30 \%$ of patients. However, it is associated with the highest rate of treatment-related mortality of all the interventions for MM. Following the introduction of new biological agents for the management of MM, the question of what role alloSCT has in MM is raised. This article aims to review where we are with alloSCT in MM, drawing from our experience thus far to plan the future role of alloSCT if we are to capitalize on a potential donor antimyeloma immune therapeutic effect.
\end{abstract}

\section{Keywords}

Allogeneic stem cell transplantation, multiple myeloma, adoptive immunotherapy, immunomodulatory agents

Disclosure: The author has no conficts of interest to declare.

Received: March 27, 2009 Accepted: June 23, 2009 DOI: 10.17925/OHR.2009.02.0.45

Correspondence: Gordon Cook, MBChB, PhD, FRCP, FRCPath, FRCPI, Director, Blood and Marrow Transplantation, St James's Institute of Oncology, Bexley Wing, St James's

University Hospital, Leeds, LS9 7TF, UK. E: Gordon.Cook@leedsth.nhs.uk

Allogeneic stem cell transplantation (alloSCT) has been utilised in the management of leukemia for several decades and has established its role in producing long-term remission. In the context of multiple myeloma (MM), alloSCT induces the highest rate of remissions, including molecular remission, resulting in long-term disease-free survival (DFS) in $<30 \%$ of patients. ${ }^{1-3}$ However, it is associated with the highest rate of treatmentrelated mortality (TRM) of all of the interventions for MM, resulting from conditioning-related end-organ damage and the unwanted immunological toxicity of graft-versus-host disease (GVHD), in addition to immunesuppression-related opportunistic infection. ${ }^{4}$

The effectiveness of alloSCT results from the combination of high-dose chemotherapy and the adoptive transfer of immune-competent donor immune-effector cells (graft-versus-myeloma [GVM] effect). The former aims to cytoreduce and is combined with autologous stem-cell rescue as first-line therapy in suitably fit patients;, 5 however, all patients ultimately relapse. The GVM immunological effect is best demonstrated by the use of donor lymphocyte infusions (DLIS) to re-induce disease responses following relapse post-alloscT. Although less potent than that seen with chronic myeloid leukemia, it significantly contributes to the DFS effect noted with alloSCT in MM. ${ }^{7.8}$ The published evidence surrounding these two main factors in alloSCT for MM suggests that they are not mutually exclusive in their contribution to long-term DFS in MM, although the relevant contribution of each to this effect remains to be clarified. There is evidence that both the method and degree of cytoreduction and the GVM effect contribute to long-term DFS in MM. As such, the challenge for transplant physicians is how to harness the GVM effect while maintaining acceptable toxicity and extending the spectrum of patients with MM who would and could benefit from allosCT.

The survival of patients with MM ranges from a few weeks to $>20$ years, but there is good evidence that the median survival of patients has increased over the last decade ${ }^{9}$ to between three and four years. This improvement is probably due to improvements in supportive care, the introduction of novel therapies, and the increased use of high-dose therapy, which has made decision-making for younger patients with a human leukocyte antigen (HLA)-matched sibling donor even more difficult. MM has a median age at presentation of approximately 70 years and only $15 \%$ of patients are $<60$ years, with the result that only a minority of patients are eligible for consideration for alloSCT, even when reducedintensity conditioning (RIC) alloSCT is included. Myeloablative alloSCT is associated with a high TRM, most likely due to a combination of factors, including advanced age of patients, myeloma-related organ damage (particularly renal impairment), disease-associated immune dysfunction, and infection risk with the effects of previous treatment. Analysis of prognostic factors is essential to compare outcomes within and between clinical trials and, in particular, to assess whether certain treatments, such as alloSCT or 'new agents,' can overcome the effect of prognostic factors that would be associated with a poor outcome with conventional therapy. The International Staging System (ISS) is considered the standard 
Table 1: Summary of Selected Reported Series of Allogeneic Stem Cell Transplantation in Multiple Myeloma

\begin{tabular}{lllllll}
\multicolumn{7}{l}{ Functional Imaging Allogeneic Stem Cell Transplantation } \\
Conditioning Regimen & $\mathrm{n}$ & $\mathrm{CR}(\%)$ & $\mathrm{TRM}(\%)$ & $\mathrm{EFS}$ & OS & Ref. \\
Cyclophosphamide/TBI & 39 & 47.2 & 31.5 & $13.3(5 \mathrm{y})$ & $28.1(5 \mathrm{y})$ & 17 \\
\hline Melphalan/TBI & 78 & 54.7 & 35.3 & $36.2(5 \mathrm{y})$ & $44.1(5 \mathrm{y})$ & 17 \\
\hline Bu/Cyclo/TBI & 15 & 53.3 & 17 & $31(6 \mathrm{y})$ & $77(6 \mathrm{y})$ & 53 \\
\hline Cyclo/TBI ( \pm idarubicin) & 53 & 19 & 34 & Med. 18m & Med. 25m & 18 \\
\hline Mel/TBI & 72 & 38 & 22 & $31.4(10 \mathrm{y})$ & $39.9(10 \mathrm{y})$ & 36 \\
\hline
\end{tabular}

Reduced-intensity Conditioning Allogeneic Stem Cell Transplantation

\begin{tabular}{|c|c|c|c|c|c|c|}
\hline Conditioning regimen & $\mathrm{n}$ & CR (\%) & TRM (\%) & EFS & OS & Ref. \\
\hline Flu/Bu/ATG & 41 & 24 & 17 & $41(2 y)$ & $62(2 \mathrm{y})$ & 20 \\
\hline $\mathrm{Flu} / \mathrm{TBI}_{200 \mathrm{~Gy}}$ & 52 & 27 & 17 & 29.4 (1.5y) & $41(1.5 y)$ & 22 \\
\hline ASCTw Flu/TBI $200 G y$ & 16 & 62 & 16 & 36 (3y) & 62 (3y) & 24 \\
\hline ASCTw Flu/Bu & 46 & 33 & 11 & - & $57(2 y)$ & 4 \\
\hline ASCT $*$ Flu/Mel/ATG & 17 & 73 & 18 & $56(2 y)$ & $74(2 y)$ & 54 \\
\hline ASCTw TBI & 54 & 57 & 7 & $55(2 y)$ & $78(2 y)$ & 19 \\
\hline ASCTw Flu/Bu/ATG & 65 & 62.2 & 10.9 & Med. 32m & Med. 35m & 23 \\
\hline ASCTw Flu/Mel/TBI2 $200 G y$ & 45 & 64 & 36 & 13 (3y) & 36 (3y) & 55 \\
\hline Non-relapse/ref. & 12 & - & - & 80 (3y) & 80 (3y) & \\
\hline
\end{tabular}

$C R=$ complete relapse; TRM = treatment-related mortality; EFS = event-free survival; OS = overall survival; $C y c l o=$ cyclophosphamide: ASCT $=$ planned tandem autologous stem cell transplant (ASCT) followed by an alloSCT; Flu = fludarabine; TBI200Gy = single fraction total body irradiation; $B u=$ intravenous busulphan; $A T G=$ antithymocyte globulin; $M e l=$ highdose melphalan; med. $=$ median; $m=$ month; $y=$ year .

prognostic model and identifies three risk categories based on levels of $\beta_{2}$-microglobulin and albumin. ${ }^{10}$ Cytogenetic and molecular genetic abnormalities have also been shown to be useful predictors of outcome, with the presence of chromosome 13 deletion- $t(4 ; 14), t(14 ; 16), t(14 ; 20)$ and deletion17p by fluorescence in situ hybridization (FISH) being associated with adverse outcome.11-13,14

Following the first alloSCT for MM in the early 1980s, a retrospective analysis of the European Group for Blood and Marrow Transplantation (EBMT) registry data with a median follow-up of 6.5 years reported that myeloablative alloSCT achieved an overall survival of $40 \%$ but was associated with a high TRM. ${ }^{15}$ A subsequent comparison of patient cohorts transplanted in 1983-1993 and 1994-1998 showed that TRM decreased from 46 to $30 \%{ }^{4}$ due to better patient selection, particularly transplantation earlier in the course of the disease. Subsequently, there were several reports from single centres indicating that with stringent patient selection, better results could be obtained (see Table 1). Two prospective, randomized studies have included a biological randomization to alloSCT. In the US Intergroup S9321 study, patients were randomized to autologous SCT, chemotherapy, or Cy/total body irradiation (TBI) ablative alloSCT if a matched sibling was identified. The alloSCT arm was closed early as a result of high TRM (53\%). However, a subsequent analysis with seven-year follow-up showed identical survival at 39\% with slightly superior progression-free survival (PFS) in the alloSCT arm (22 versus 15\%). There was also evidence of a plateau on the survival curve of the alloSCT arm. ${ }^{16}$ Similar results were obtained from a retrospective British Society of Blood and Marrow Transplantation (BSBMT) study of 139 patients $<55$ years of age, which showed superiority for melphalan/TBI over cyclophosphamide/ TBI conditioning in both TRM and relapse rates. ${ }^{17}$ In an attempt to reduce TRM and the high incidence of GVHD, patients $<55$ years of age entered into the Hemato-Oncology Cooperative Group (HOVON) 24 MM study who had an HLA-matched sibling received a Cy/TBI T-cell depleted (TCD)
alloSCT. ${ }^{18}$ Patients without a donor were randomized to receive a Cy/TBI autologous stem cell transplant (ASCT) or no further chemotherapy. Despite TCD, the TRM remained high at 34\%, and there was a high relapse rate with only three out of 53 alloSCT recipients in complete relapse (CR) at a median follow-up of 38 months. The results of this study suggest that profound transcranial Doppler (TCD) should be avoided.

As a result of the experience of full-intensity alloSCT, investigators explored the use of RIC alloSCT in MM to reduce TRM and to permit the application of alloSCT to older patients. Several studies have shown that this approach is feasible, with a significant reduction in TRM (see Table 1). By definition, in the absence of intensive chemo-radiotherapy, RIC alloSCT is to a large extent dependent on allogeneic GVM effects. ${ }^{19}$ A number of phase II studies have reported similar findings. ${ }^{20-22}$ In these studies, the presence of chronic GVHD was associated with the achievement of CR and improved overall survival (OS)/PFS.

Two prospective 'biologically randomized' studies of RIC alloSCT have been published. IFM-99-03/04 enrolled patients with poor-risk disease as defined by the presence of de/13 by FISH along with elevated $\beta_{2}$-microglobulin $(>3 \mathrm{mg} / \mathrm{I}){ }^{23}$ Those without a sibling donor received tandem ASCT, whereas those with a sibling donor received a sequential ASCT/RIC alloSCT, the latter conditioning utilizing antithymocyte globulin (ATG) (Genzyme). This study showed no benefit in terms of OS/event-free survival (EFS) and no patients in either arm achieved durable EFS. Bruno et al. reported on a group of unselected patients who were biologically randomized in a similar fashion to tandem ASCT or a sequential ASCT/RIC alloSCT. ${ }^{24}$ The RIC alloSCT arm demonstrated significantly superior OS/EFS. While no prospective trials have compared full-intensity with RIC alloSCT in this setting, an EBMT analysis has shown similar OS with both approaches. As might be expected, RIC alloSCT had a lower TRM but a higher relapse rate and lower PFS. ${ }^{25}$

Generally speaking, outcomes with matched unrelated donor (MUD) alloSCT have improved with time and in many settings have become equivalent to matched sibling transplantation. However, retrospective studies in myeloma have shown a significantly higher TRM than sibling alloSCT, ${ }^{26}$ and myeloablative MUD alloSCT is not currently recommended and should be carried out only in the context of prospective clinical trials. However, the role of RIC MUD allOSCT remains to be defined. Encouraging results have been reported for TRM of approximately $20-21 \%$ at two and three years. ${ }^{27,28}$ Clearly, further prospective trials are warranted in order to better define the role of RIC MUD alloSCT for patients with myeloma.

No prospective trials have compared functional imaging (FI) with RIC alloSCT in MM, and the type of RIC regimen and the therapy previously administered may matter. However, an EBMT analysis has shown similar OS with both approaches, although the RIC alloSCT group had a lower TRM but a higher relapse rate and thus lower PFS. ${ }^{25}$ This may reflect the heterogeneity of the patient populations, and further adaptation of RIC alloSCT may be required to maximize the antitumor effect in association with the well-established reduced TRM. In patients over 40 years of age or in those deemed unfit for an FI alloSCT, RIC alloSCT has been shown to reduce TRM. By definition, as FI conditioning is not given, RIC transplants are largely dependent on the adoptive immunotherapeutic effect of GVM. As such, a 'tandem' sequential strategy has been developed to generate a 
minimal tumor burden pre-RIC alloSCT using high-dose melphalanconditioned ASCT. A number of phase II studies have reported the feasibility and efficacy of such a strategy, especially in association with limited chronic GVHD. ${ }^{20,21,25,29}$ Bruno and colleagues reported on a 'biologically randomized' study of sequential ASCT/RIC alloSCT compared with double ASCT, demonstrating a superior OS and EFS with the RIC alloSCT arm. ${ }^{24}$ Two further studies are yet to be reported (EBMT NMAM2000, where a tandem ASCT followed by a MRD RIC alloSCT is compared with double ASCT, and the US Intergroup study). Therefore, in patients deemed to have an adequate performance status/comorbidity profile, sequential ASCT and RIC alloSCT may be a suitable therapeutic strategy to induce a minimal residual disease (MRD) state (ASCT) on which to build the adoptive immunotherapeutic effect of GVM (RIC alloSCT).

In light of the limitations in availability of a matched related (family) donor, alternate sourcing of donor stem cell grafts is routinely employed in the management of acute and chronic myeloid malignancies. The use of matched volunteer-unrelated donor (VUD) grafts utilizing FI conditioning is not generally supported even in younger patients in the first treatment phase. The improvement in VUD FI alloSCT outcomes represents a future development, but currently should be considered only as part of a clinical trial protocol. ${ }^{26}$ The role of RIC MUD alloSCT remains to be defined, although recent results have reported TRM of approximately $20 \%{ }^{26,27}$ Clearly, further prospective trials are warranted in order to better define the role of RIC MUD alloSCT for patients in the first treatment phase for myeloma. Similarly, the use of umbilical cord stem cells as a source for GVM has been reported in a limited number of case reports but only in patients with advanced disease,,$^{30,31}$ and again should be explored only as part of a clinical study protocol.

The treatment decision-making process involving the use of alloSCT in the overall management strategy of patients with MM represents some of the most difficult concerns hemato-oncology patients face. Patients need to be fully informed of the risks and potential benefits of undergoing such a treatment, and the clinical treatment strategy that is adopted should represent both the clinical evidence and the patient's understanding and informed consent. The use of pre-transplant comorbidity scoring systems has evolved in the stem cell transplant field, although these have yet to be validated in European populations and, specifically, their utility in a highrisk patient population such as $\mathrm{MM}$ remains to be determined. ${ }^{32,33} \mathrm{~A}$ potential clinical decision-making tree is represented by the flow diagram illustrated in Figure 1. Patients $<40$ years of age with a matched sibling donor should be considered suitable for FI alloSCT in the first treatment phase. Patients between 40 and 50 years of age may be considered suitable for FI MRD alloSCT based on biological performance status, comorbidity, and disease-related features at the discretion of the transplant physician. The optimal conditioning regimen has never been prospectively compared, but retrospective analyses favor melphalan $140 \mathrm{mg} / \mathrm{m}^{2}$ with fractionated TBI (12Gy in six fractions). ${ }^{17}$

Is alloSCT the final therapeutic intervention for MM? Experience suggests that this in fact is more of a therapeutic platform on which to build a strategy for long-term DFS. Donor lymphocyte infusions from the original donor of the allograft are given for three main reasons: for relapse (which may be at various levels), pre-emptively in patients at high risk for relapse, and for correction of mixed chimerism in order to preserve a GVM effect
Figure 1: Decision Flow Diagram for Allogeneic Stem Cell Transplantation in Myeloma

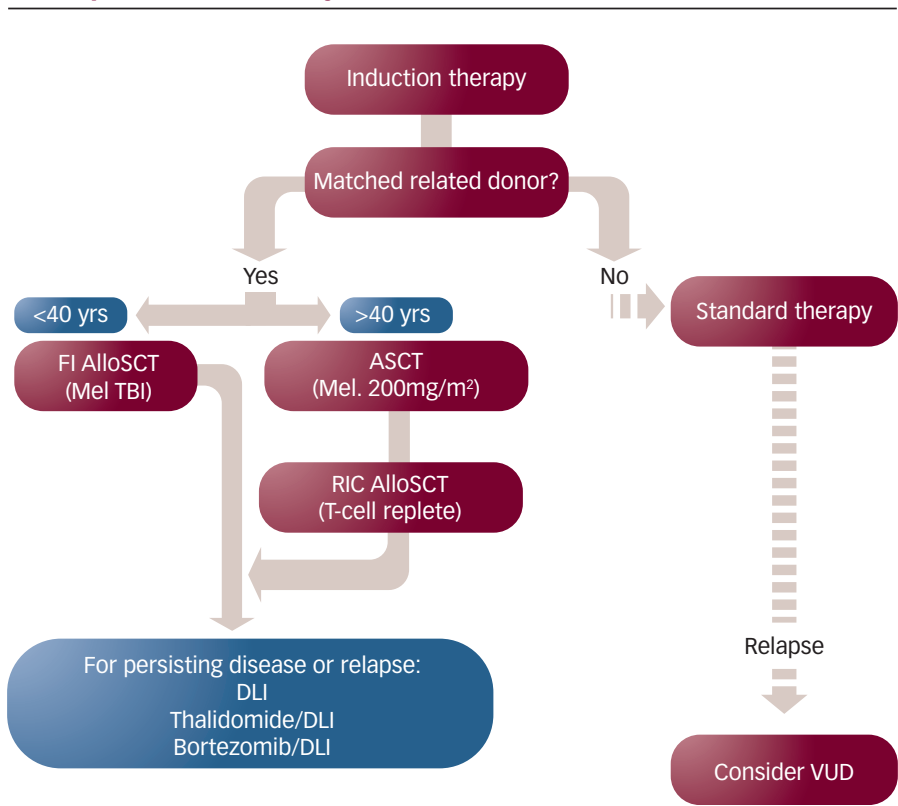

FI alloSCT = full-intensity allogeneic stem cell transplantation; RIC alloSCT = reduced-intensity alloSCT; Mel. = high-dose melphalan; $T B I=$ fractionated total body irradiation; $A S C T=$ planned tandem autologous stem cell transplant; $D L I=$ donor lymphocyte infusions; $V U D=$ volunteerunrelated donor alloSCT.

and reduce the chance of relapse. There has been little work aimed at defining the main effector cells (T cells or natural killer [NK] cells) and may in fact vary from disease to disease. Little is known about the main 'antigenic' targets and in MM many immunotherapy trials have focused on the MM-specific idiotypic determinant of the immunoglobulin (Ig) variable region, although other antigenic determinants have been suggested, such as the cancer germline antigens. ${ }^{34,35}$ The available evidence suggests that the GVM effect is 'moderate' and that high doses of DLI resulting in clinical acute and chronic GVHD are required to achieve sustained disease responses. Disease responses occur in up to half of relapsed patients and up to $20 \%$ achieve CR, although the median PFS can be short. ${ }^{7,36}$ Zeiser and colleagues reviewed the published myeloma donor lymphocyte infusion (DLI) data in 2004. ${ }^{37}$ Overall, 40-52\% responded, with 25\% having a CR. Response durations ranged from six to 15 months and responses were mainly seen after multiple DLI with high T-cell doses. Acute and chronic GVHD were seen in half and one-third, respectively. Attempts to separate the GVM and GVHD effects have been made using CD8-depleted DLI. ${ }^{38}$ Only one of nine patients who received $3 \times 10^{7} \mathrm{CD}^{+}$cells/kg DLI had GVHD. Disease response data in patients with $\mathrm{MM}$ using this approach are lacking, and there will be concern that reducing GVHD will also result in fewer patients responding.

There are few reports about the use of DLI to prevent relapse (in the absence of mixed chimerism) and also few data about its use for eliminating low-level MRD detected by molecular methods. Prophylactic DLI would be expected to have substantial toxicity; however, in the setting of RIC alloSCT, the use of DLI is quite different. When these regimens involve in vivo T-cell depletion using alemtuzamab or ATG, mixed chimerism is not an uncommon outcome, and may be associated with an increased risk for relapse. The University College London group reported 109 DLIs in 46 patients (including 19 MM patients) and a chimeric response 
in 30 of 35 patients. ${ }^{39}$ GVHD was observed in 12 patients. Disease responses occurred in nearly two-thirds of MM patients, although surprisingly they were not necessarily associated with chimeric responses. However, much investigation is required before we can confidently advise about the dose and timing of DLI, and each disease is likely to be different., ${ }^{40,41}$ Kröger expresses the view that we should be targeting molecular remission and not settling for less rigorous assessments of disease..$^{42}$ The shortcomings of these data have been noted (heterogeneity and short follow-up), and disease-specific clinical trials are required.

With the expansion of available biological therapies in the management of $\mathrm{MM}^{43}$ the rationale for using such therapies post-alloSCT has been proposed, either pre-emptively or to treat relapsing disease. ${ }^{42}$ There are limited data concerning the use of bortezomib in this setting. In a small study, two cycles of four doses were completed by 14 patients, ${ }^{44}$ with neurotoxicity and thrombocytopenia being the most frequent reported adverse events, and in four patients there may have been some stimulation of GVHD. Nonetheless, complete and partial response rates of 30 and $50 \%$ were promising outcomes. An Italian series of 23 patients reported similar results, with $61 \%$ response and $22 \%$ immunofixationnegative CRS with PFS of six months, although similar toxicities were observed in nearly a half. ${ }^{45}$ Sixty percent of these patients also received steroids, but this did not affect response.

There are small series reporting the use of thalidomide post-alloSCT. The InterGroupe Francophone du Myélome (IFM) group reported a 29\% response rate with acceptable toxicity. ${ }^{46}$ Kröger and colleagues reported a $67 \%$ response rate with a $84 \%$ two-year $\mathrm{PFS}{ }^{47}$ Thalidomide has been used in several studies as a maintenance strategy post-alloSCT. ${ }^{48-50}$ Developed as an analog of thalidomide with immunomodulatory effects, lenalidomide has been shown to be efficacious in the management of MM. ${ }^{51,52}$ However, there are fewer data concerning the use of lenalidomide in this situation. Studies are ongoing to determine the effect of post-alloSCT administration of lenalidomide either as pre-emptive therapy or for early relapsing disease, and the efficacy of this strategy will be as important as the drug-related adverse events, especially the effect, if any, on GVHD.

Although much has been learned by experience to date in the use of alloSCT in the long-term management of MM, its role is less certain than in other hematological malignancies. The overarching premises of the putative GVM effect still remain elusive without considerable risk to the recipient. Nonetheless, with all other therapy MM is a universally fatal malignancy, and while improvements have been made in OS with the introduction of new drugs, the median survival is still unacceptably short. With the introduction of novel agents, the potential to take patients to alloSCT with considerably reduced tumor burdens represents the way forward, while permitting a more intensive conditioning regimen (Mini, Midi, Maxi) ${ }^{42}$ resulting from reduced pretransplant end-organ therapy-related damage. Furthermore, the alloSCT should be viewed as the platform from which to launch a pre-emptive consolidative strategy to minimise the risk for disease relapse. This remains the challenge of the imminent future for the latter-day pioneers of alloSCT medical practice.
1. Corradini P, Voena C, Tarella C, et al., J Clin Oncol, 1999;17(1):208-15.

2. Bensinger WI, Clin Adv Hematol Oncol, 2004;2(1):46-52.

3. Rabitsch W, Prinz E, Ackermann J, et al., Eur J Haematol, 2004;72(1):26-31.

4. Gahrton G, Svensson H, Cavo M, et al., Br J Haematol, 2001;113(1):209-16

5. Attal M, Harousseau JL, Facon T, et al., N Eng/ J Med, 2003;349(26):2495-2502.

6. Child JA, Morgan GJ, Davies FE, et al., N Eng/ J Med, 2003:348(19):1875-83.

7. Lokhorst HM, Wu K, Verdonck LF, et al., Blood, 2004;103(11):4362-4

8. Salama M, Nevill T, Marcellus D, et al., Bone Marrow Transplant, 2000:26(11):1179-84.

9. Kumar SK, Rajkumar SV, Dispenzieri A, et al., Blood, 2008;111(5):2516-20.

10. Greipp PR, San Miguel J, Durie BG, et al., J Clin Oncol, 2005:23(15):3412-20.

11. Chiecchio L, Protheroe RK, Ibrahim AH, et al., Leukemia, 2006;20(9):1610-17.

12. Gutierrez NC, Castellanos MV, Martin ML, et al., Leukemia, 2007;21(1):143-50.

13. Avet-Loiseau H, Attal M, Moreau P, et al., Blood, 2007;109(8):3489-95.

14. Shaughnessy JD Jr, Zhan F, Burington BE, et al., Blood, 2007;109(6):2276-84.

15. Gahrton G, Tura S, Ljungman P, et al., Bone Marrow Transplant, 1991;7(Suppl. 2):32.

16. Barlogie B, Zangari M, Bolejack V, et al., Clin Lymphoma Myeloma, 2006;6(6):469-74.

17. Hunter HM, Peggs $K$, Powles R, et al., Br J Haematol, 2005;128(4):496-502.

18. Lokhorst HM, Segeren CM, Verdonck LF, et al., J Clin Oncol, 2003;21(9):1728-33.

19. Maloney DG, Molina AJ, Sahebi F, et al., Blood,
2003;102(9):3447-54.

20. Mohty M, Boiron JM, Damaj G, et al., Bone Marrow Transplant, 2004;34(1):77-84

21. Perez-Simon JA, Caballero D, Mateos MV, San Miguel JF, Leuk Lymphoma, 2004;45(9):1725-9.

22. Gerull $\mathrm{S}$, Goerner $\mathrm{M}$, Benner $\mathrm{A}$, et al., Bone Marrow Transplant, 2005; 36(11):963-9.

23. Frederic Garban MA, Michallet M, Hulin C, et al., Blood, 2006;107(9):3474-80

24. Bruno B, Rotta M, Patriarca F, et al., N Eng/ I Med 2007:356(11):1110-20.

25. Crawley C, lacobelli S, Bjorkstrand B, et al., Blood, 2007:109(8):3588-94.

26. Shaw BE, Peggs $K$, Bird JM, et al., Br J Haematol, 2003:123(5):886-95

27. Kroger N, Sayer HG, Schwerdtfeger R, et al., Blood, 2002;100(12):3919-24

28. Bruno B, Sorasio R, Patriarca F, et al., Eur J Haematol, 2007;78(4):330-37.

29. Alyea E, Weller E, Schlossman R, et al., Bone Marrow Transplant, 2003;32(12):1145-51.

30. Fenk R, Neumann F, Fenk B, et al., Leuk Res, 2008;32(7):1157-9.

31. Ando T, Yujiri T, Tominaga T, et al., Eur J Haematol, 2005;74(2):175-9.

32. Sorror ML, Maris MB, Storb R, et al., Blood, 2005;106(8):2912-19.

33. Parimon T, Au DH, Martin PJ, Chien JW, Ann Intern Med, 2006:144(6):407-14

34. Goodyear O, Piper K, Khan N, et al., Blood, 2005:106(13):4217-24

35. Atanackovic D, Arfsten J, Cao Y, et al., Blood, 2007:109(3):1103-12.

36. Kuruvilla J, Shepherd JD, Sutherland HJ, et al., Biol Blood Marrow Transplant, 2007;13(8):925-31.

37. Zeiser R, Bertz H, Spyridonidis A, et al., Bone Marrow Transplant,
2004;34(11):923-8

38. Alyea EP, Canning C, Neuberg D, et al., Bone Marrow Transplant, 2004;34(2):123-8

39. Peggs KS, Thomson $\mathrm{K}$, Hart DP, et al., Blood, 2004;103(4):1548-56

40. Peggs K, Mackinnon S, Leukemia, 2004;18(9):1541-2, author reply 1542-3.

41. Marks DI, Lush R, Cavenagh J, et al., Blood, 2002;100(9):3108-14.

42. Kroger N, Mini-Midi-Maxi? Leukemia, 2007;21(9):1851-8

43. Mitsiades CS, Hayden PJ, Anderson KC, Richardson PG, Best Pract Res Clin Haematol, 2007;20(4):797-816.

44. van de Donk NW, Kroger N, Hegenbart U, et al., Bone Marrow Transplant, 2006;37(12):1135-41.

45. Bruno B, Patriarca F, Sorasio R, et al., Haematologica, 2006;91(6):837-9

46. Mohty M, Attal M, Marit G, et al., Bone Marrow Transplant, 2005;35(2):165-9.

47. Kroger N, Shimoni A, Zagrivnaja M, et al., Blood, 2004;104(10):3361-3.

48. Attal M, Harousseau JL, Leyvraz S, et al., Blood, 2006;108(10):3289-94.

49. Brinker BT, Waller EK, Leong T, et al., Cancer, 2006;106(10):2171-80.

50. Feyler S, Rawstron A, Jackson G, et al., Br J Haematol, 2007;139(3):429-33

51. Reddy N, Hernandez-llizaliturri FJ, Deeb G, et al., Br J Haematol, 2008;140(1):36-45

52. Weber DM, Chen C, Niesvizky R, et al., N Engl J Med, 2007;357(21):2133-42.

53. Kroger $\mathrm{N}$, Einsele $\mathrm{H}$, Wolff $\mathrm{D}$, et al., Bone Marrow Transplant, 2003; 31(11):973-9.

54. Kroger N, Schwerdtfeger R, Kiehl M, et al., Blood, 2002;100(3):755-60.

55. Lee CK, Badros A, Barlogie B, et al., Exp Hematol, 2003:31(1):73-80 